
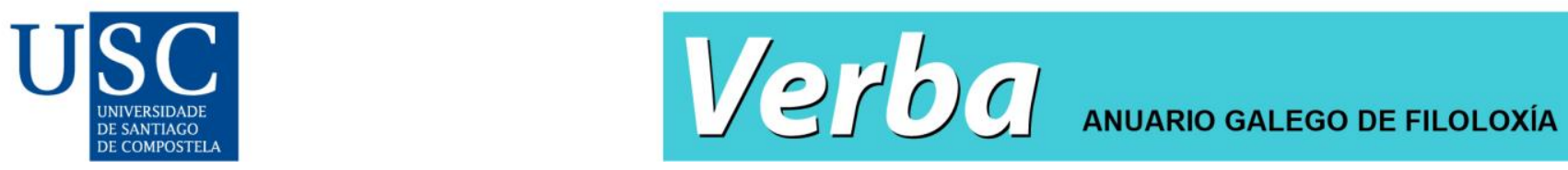

Verba: Anuario Galego de Filoloxía, 48, 2021. ISSN: 2174-4017

https://doi.org/10.15304/verba.48.7398

Revista de libros

\title{
Yeray González Plasencia (2019): Comunicación intercultural en la enseñanza de lenguas extranjeras. Berlín: Peter Lang, 356 pp.
}

\author{
Raúl Urbina Fonturbel ${ }^{1}$ \\ 1Universidad de Burgos, España
}

Esta obra de Yeray Gómez Plasencia, que tiene como origen la tesis doctoral realizada en la Universidad de Salamanca dirigida por Noemí Domínguez García, realiza una aportación interesante y muy necesaria a la comunicación de los aspectos interculturales en la enseñanza de lenguas extranjeras. Partiendo de aspectos simbólicos, dinámicos y humanos de la cultura, estudia los procesos de comunicación en comunicantes con distintas identidades culturales. La obra no supone solo un análisis exhaustivo de la comunicación intercultural, sino que, con un enfoque didáctico, se propone llevar a la práctica docente esta perspectiva.

Desde su nacimiento a mediados del siglo XX, la comunicación intercultural no solo ha pasado a convertirse en una disciplina independiente, sino en objeto de estudio de saberes que van desde la antropología, la sociología, la lingüística a las relaciones internacionales o a la educación. El propósito de esta obra es analizar la comunicación intercultural en su dimensión lingüística y educativa aplicada a la enseñanza de lenguas extranjeras en su aspecto competencial. Para facilitar la lectura a investigadores con distinto tipo de intereses, aunque cada apartado del libro está relacionado con los demás, se articula también de manera independiente.

El autor analiza tanto los modelos teóricos como la aplicación de la Competencia Comunicativa Intercultural (CCI). El libro está estructurado con una oportuna secuencia progresiva. En su aspecto más teórico, empieza con la aproximación al concepto de cultura, luego pasa al análisis de la comunicación intercultural, para finalizar estudiando la competencia comunicativa intercultural. La dimensión práctica del trabajo se centra en el repaso de las herramientas de medición más conocidas en el campo de la comunicación intercultural (CCI) y finaliza ofreciendo a los investigadores un interesante y útil instrumento de medición, como es la Escala de Recursos Interculturales (ERI).

En ese binomio esencial que configuran los aspectos lingüísticos y los culturales, la comunicación intercultural supone uno de los aspectos básicos en la enseñanza de una lengua extrajeras (LE). Aunque los documentos de referencia en la enseñanza de lenguas incorporan de 
manera significativa la comunicación intercultural, se hace necesario $-\mathrm{y}$ es uno de los cometidos principales de la presente obra - ahondar en los componentes que comprende la CCI, la identificación de los factores que resultan eficaces para la comunicación intercultural y la sustitución de un enfoque de mera transmisión de saberes de la lengua meta con una valencia meramente informativa por una perspectiva formativa que desarrolle la conciencia crítica de los aprendices.

El capítulo 1 está dedicado a realizar una aproximación al concepto de cultura. En un campo inicialmente puede parecer difuso y con enfoques muy dispares, se hace indispensable realizar una delimitación y un análisis del término cultura para llegar a los aspectos que resultan relevantes para el propósito de esta obra y que resulte pertinente para la enseñanza de las lenguas extranjeras. El capítulo se inicia abordando los distintos enfoques antropológicos (evolucionismo, particularismo histórico, lo superorgánico, el configuracionismo, el funcionalismo, el neoevolucionismo, el cognitvismo, el materialismo cultural y la antropología simbólica) del término para luego tratar otros enfoques interpretativos, como los adaptacionistas e idealistas, y acabar explicando el concepto de cultura en la enseñanza de las lenguas extranjeras. Este recorrido por todos los enfoques de la cultura será de gran utilidad para que los docentes de LE sepan escoger con acierto su manera de enfocar su práctica docente de la cultura.

Bajo la premisa de que lengua y cultura son indisociables, el reto para los docentes de lenguas extranjeras es integrar de modo adecuado la cultura en la enseñanza de lenguas. No se trata tanto de centrarse en el concepto mismo de cultura, sino sobre la manera que tiene de construirse la cultura en la vida diaria. En este sentido, resulta importante la manera en que se relacionan mutuamente cultura y sociedad. Para construir estas realidades culturales, los individuos aplican la competencia cultural y la ejecutan en función del contexto y de sus condiciones individuales. Pero, en una situación de interculturalidad, se hace necesario saber cómo conciliar esas dos competencias - competencia comunicativa y competencia intercultural- que "chocan". Para resolver esta cuestión, es necesario partir del análisis del contexto de interacción y no centrarse solamente en los grupos o en los individuos. De esta manera, Gómez Plasencia concibe la cultura como "los significados, modulados por las expectativas que posee el individuo fruto de su proceso de socialización y de experiencias anteriores, construidos durante la interacción de una persona con otros individuos o con el entorno" (p. 76). De esta manera de concebir la cultura se desprenden tres características: es simbólica, en cuanto no atiende a repertorios de características de una comunidad, sino a los significados concretos que le atribuyen los individuos cuando interactúan con esa realidad; es dinámica, puesto que los significados se construyen durante la interacción y, en virtud de esa construcción interactiva, se basa en significados concretos y no en generalidades; por último, es humana, porque no interactúan "las culturas", sino las personas: lo social parte de los individuos, que son los que asignan y construyen los significados culturales. De esta manera de concebir la cultura se derivan implicaciones concretas para la enseñanza de las lenguas extranjeras, entre las que destaca el papel del instructor como coadyuvante a la creación de significados y cuya labor, en consecuencia, no tendría que consistir en transmitir información sobre la cultura meta, sino en procurar que los aprendices interpreten críticamente otras realidades. Para dar este paso, resulta indispensable que el docente cuestione su visión de cultura y que sea consciente de que está transmitiendo solo un aspecto que posee, en sí mismo, múltiples interpretaciones posibles. Esta manera de enfocar la enseñanza de la cultura contribuye, de igual modo, al empoderamiento del aprendiz en este proceso de interacción comunicativa que nunca es neutro, sino abierto al juicio crítico. 
El segundo capítulo se centra en la comunicación intercultural y evidencia de manera muy acertada que su estudio reviste cierta complejidad. Comienza con las contribuciones del relativismo cultural y lingüístico, de la etnografía de la comunicación y de la pragmática intercultural para, describir, a continuación, las diferencias de las culturas desde un enfoque comparativo y universal, opuestas al enfoque interactivo, que atiende más a la comunicación de individuos que se perciben como diferentes y que interactúan en un contexto concreto. Especialmente útil resulta uno de los apartados, dedicado a elementos como la identidad, la ansiedad, la incertidumbre, las estrategias comunicativas o los procesos de adaptación. El capítulo finaliza con un resumen de las principales aportaciones de ángulos tan diversos para la CCI como los que acabamos de reseñar.

Partiendo de una experiencia personal, Yeray González Plasencia constata que diferentes culturas pueden convivir en un mismo entorno y que los patrones interculturales se interpretan y se construyen gracias a los significados que comparte una colectividad. Se trata, en suma, de ir estudiando todas las vertientes teóricas para indagar qué pueden aportar los estudios de comunicación intercultural a la enseñanza de las lenguas extranjeras. Las teorías del relativismo lingüístico y cultural, que manifiestan la tensión entre lo universal y lo particular, constituyen la piedra angular de los enfoques sobre comunicación intercultural. A continuación, se exponen los principales enfoques sobre la variación cultural con el fin de establecer una distinción entre las aproximaciones transculturales y las aproximaciones interculturales. También se estudian las dimensiones que son universales a todas las culturas teniendo en cuenta que no en todas las sociedades se manifiestan con la misma intensidad o frecuencia. Se analiza también la comunicación intercultural desde la perspectiva interactiva de personas que perciben sus identidades culturales como algo diferente. Para ello, el autor ha escogido ilustrar estos aspectos con las estrategias comunicativas en la negociación de un conflicto, los factores que afectan a la comunicación afectiva, la gestión de la identidad y el proceso de aculturación y adaptación. Aunque existan diferencias, instructores y aprendices tienen que ir tomando consciencia no solo de lo que los diferencia, sino de todos aquellos aspectos que comparten y que son comunes para ambos. Este capítulo contiene reflexiones de mucho interés sobre la delimitación de cultura e identidad, que tiene en cuenta que no todas las identidades pasan necesariamente por ser culturales. Un docente de lengua extranjera no solamente transmite un código de comunicación, sino que, por medio de su labor, también puede legitimar o deslegitimar determinadas posiciones. En este sentido, la cultura implica una visión del mundo y el docente puede contribuir a valor la realidad y construirla. Por todo ello, es preciso intentar dar cabida a interpretaciones diferentes y no crear una visión monolítica de la cultura. Por esa razón, resulta esencial trabajar de modo adecuado las estrategias en la comunicación intercultural.

El tercer capítulo está dedicado a la competencia comunicativa intercultural y, por tanto, a la utilidad de la CCI para la enseñanza de lenguas extranjeras. Empieza por estudiar el proceso paulatino de separación y diferenciación de la competencia intercultural como competencia diferenciada de la competencia comunicativa. A continuación, se abordan los campos que estudia la CCI y las subsiguientes implicaciones para la enseñanza de lenguas. Más adelante, se disecciona la estructura de la CCI partiendo de los modelos más representativos y también, más adelante, se analiza el tratamiento que dan a la CCI los documentos como el MCER (Marco Común de Referencia para la Enseñanza de las Lenguas) del Consejo de Europa y el PCIC (Plan Curricular del Instituto Cervantes) para la enseñanza del español como lengua extranjera. 
En la actualidad, relacionarse con individuos de otras culturas resulta algo habitual. En esa relación, conviene preguntarse qué marcos de referencia aplican los individuos, qué aspectos comparten y con cuáles se diferencian. La CCI supone un instrumento sólido para abordar todas estas cuestiones. Todos nosotros, en cuanto individuos, poseemos una competencia cultural y una ejecución de la misma que, en ocasiones, obstaculiza la asimilación adecuada de una cultura de una segunda lengua. Por lo tanto, no solo es importante adquirir el sistema lingüístico y gramatical de una segunda lengua, sino que, para desenvolverse de manera adecuada en un entorno cultural que no es el propio, es necesario activar nuestra CCI mediante recursos afectivos, cognitivos y procedimentales adecuados. Del mismo modo que el componente intercultural no es equivalente al componente cultural, la competencia de comunicación intercultural no es equiparable, sin más, al componente cultural. De igual modo, tampoco es útil la reducción a contrastes muy generales y operativos que conducen a la legitimación de los estereotipos y el etnocentrismo, como ocurre cuando trabajamos con conceptos como la "nacionalidad". Aquí, el autor se plantea qué atributos y destrezas garantizan el éxito en la interacción intercultural y cómo pueden ser desarrollados y medidos. A continuación, se analiza la noción de CCI desde la relación entre lengua y cultura en la enseñanza de una lengua extranjera, la definición teórica de dicha competencia y las implicaciones que tienen en los enfoques interculturales. Se revisan los principales modelos de la CCI y, por último, se analiza cómo ha sido abordada dicha competencia en los documentos de referencia para la enseñanza del español como lengua extranjera más recientes.

La CCI supone tanto la necesidad de activación y movilización de determinados recursos como la valoración que efectúan los participantes sobre los mismos. En cuanto al primer aspecto, la CCI debe tener en cuenta las dimensiones afectiva, cognitiva y procedimental, por lo que es necesario movilizar actitudes, conocimientos y destrezas. Como se sostiene en varios lugares a lo largo de la obra, no se trata de la mera acumulación de saberes factuales. La interacción en la CCI tiene lugar entre individuos y no entre culturas. La identidad cultural es solo una de las identidades que poseen los individuos. En suma, la CCI, tal y como se ha apuntado, no se refiere tanto a culturas como a personas. La interacción misma implica unos efectos. El autor asume que no existen recursos específicos para la creación de la conciencia crítica, sino que de alguno de los recursos afectivos, cognitivos y procedimentales se deriva un componente crítico. La adquisición de la CCI no es tanto una cuestión de "enseñanza" como de asimilación y desarrollo por medio de experiencias vividas por los individuos.

El capítulo cuarto está dedicado a la medición de la Competencia Comunicativa Intercultural. En primer lugar, se realiza un repaso a las diferentes maneras de medir la CCI intercultural, para acabar ofreciendo una nueva herramienta que permite una medición más efectiva en la enseñanza de lenguas extranjeras: la Escala de Recursos Interculturales (ERI). En segundo lugar, se describen las cuatro fases de elaboración de la ERI.

Se realiza una revisión del concepto de "medición" en la CCI y se enfoca la diferencia entre "medir" y "evaluar". A continuación, de describen los instrumentos en el ámbito e la interculturalidad y se valora su posible aplicación a la CCI. Por último, la ERI se dibuja como un cuestionario destinado a cubrir una medición adecuada de la CCI. La ERI se ha configurado siguiendo un proceso en cuatro fases: descripción de las áreas principales de la CCI, definición de los ítems, redacción de las instrucciones y realización de una prueba piloto. En la descripción de las principales áreas de la CCI, se atiende a una clasificación apriorística que comprende los componentes de cada una de las dimensiones de esta competencia. De este modo, el autor, dentro 
de las estrategias, incluye la gestión de la interacción, la gestión de la comunicación, el análisis y planificación y la interpretación de otras realidades; en las actitudes, contempla la apertura, la autoconfianza, el respecto, la disposición y la empatía; y, en lo que se refiere a los conocimientos, abarca la cultura material, la cultura comunicativa y la identidad cultural. Por lo que a la definición de los ítems se refiere, los criterios que han de cumplir son que impliquen interacción, que reflejen exclusivamente una de las áreas propuestas en la estructura anterior, que se definan de un modo suficientemente general y que no incluyan sesgos culturales y lingüísticos. Una vez realizado los ajustes previos pertinentes, la prueba piloto sirvió para realizar una adaptación que superase las dificultades detectadas constituida por la versión final, que consta de 97 ítems.

En definitiva, el libro de Yeray González Plasencia supone un acercamiento de enorme interés tanto en el campo teórico de la Comunicación Intercultural y a la Competencia Comunicativa Intercultural como en su aplicación práctica a la enseñanza de las lenguas extranjeras. La obra consigue profundizar y conciliar de manera inteligente estas dos dimensiones y evidencia la pertinencia de atender de estudiar forma adecuada la CCI. González Plasencia propone una nueva manera de enseñar esta competencia en el aula de lenguas extranjeras: el foco no ha de estar centrado, sin más, en la cultura meta, sino también en la identidad del estudiante. Por otro lado, el objetivo no ha de estar centrado en la acumulación de saberes, sino en la conciencia crítica intercultural. Por último, por lo que respecta al docente, este no ha de ser un mero transmisor de información, sino ha de ser "cocreador" y "cointérprete" de los significados. 